\title{
Does ultrasound-guided facet joint injection reduce pain and improve mobility in patients with failed back surgery syndrome?
}

\author{
Musa Çırak, MD'1D, Sibel Çağlar Okur, MD²D \\ ${ }^{1}$ Department of Neurosurgery, Bakırköy Dr. Sadi Konuk Training and Research Hospital, Istanbul, Turkey \\ ${ }^{2}$ Department of Physical Medicine and Rehabilitation, Bakırköy Dr. Sadi Konuk Training and Research Hospital, Istanbul, Turkey
}

Lumbar disc herniation (LDH) has a gradual increase due to aging of population and lifestyle changes. Lumbar discectomy is the standard surgery for the treatment of $\mathrm{LDH}$ for more than eight decades. ${ }^{[1]}$ Despite refinements in surgical technique, ongoing back pain is still an important complication of the surgery. ${ }^{[2-4]}$ Persistent, longstanding back and/or leg pain after completed one or more lumbar disc surgery is referred to as failed back surgery syndrome (FBSS) (or postlumbar surgery syndrome/postoperative persistent pain syndrome). The exact definition of FBSS of The International Association for the Study of Pain is "Lumbar spinal pain of unknown origin either persisting despite surgical intervention or appearing after surgical intervention for spinal pain originally in the same topographical location." ${ }^{\prime[5]}$ Although failed back surgery implies any level of spine, the majority of studies reported data about lumbosacral region. ${ }^{[6]}$

Received: April 25, 2020

Accepted: Jue 22, 2020

Published online: September 11, 2020

Correspondence: Musa Cırak, MD. Bakırköy Dr. Sadi Konuk Eğitim ve Araştırma Hastanesi Nöroşirurji Kliniği, 34147 Bakırköy, İstanbul, Türkiye.

E-mail: musacirak@hotmail.com

Doi: $10.5606 /$ ehc. 2020.75727

Citation: Clrak M, Cağlar Okur S. Does ultrasound-guided facet joint injection reduce pain and improve mobility in patients with failed back surgery syndrome?. Jt Dis Relat Surg 2020;31(3):564-570.

(02020 All right reserved by the Turkish Joint Diseases Foundation

This is an open access article under the terms of the Creative Commons Attribution-NonCommercial License, which permits use, distribution and reproduction in any medium, provided the original work is properly cited and is not used for commercial purposes (http://creativecommons.org/licenses/by-nc/4.0/).

\section{ABSTRACT}

Objectives: This study aims to evaluate whether ultrasound (US)guided facet joint injection reduces pain and improves mobility in patients with failed back surgery syndrome (FBSS).

Patients and methods: This retrospective study included 27 patients ( 8 males, 19 females; mean age $43.6 \pm 11.5$ years; range, 31 to 54 years) who underwent US-guided facet injections for FBSS between January 2017 and January 2019. Patients' medial records were assessed. Pain rating scores were evaluated with the Numeric Rating Scale (NRS). Functional status was assessed with Oswestry Disability Index (ODI) version 2.0. Lumbar flexion degree was noted. After injection (lidocaine-betamethasone mixture), patients were reevaluated at first and sixth months. The study data were analyzed with Wilcoxon signed-rank test.

Results: Successful facet joint injections were achieved without any complications in all patients. The median duration of time after surgery was $7.8 \pm 1.3$ months. Mean duration of pain was $6.7 \pm 5.7$ months. There was a significant decrease in NRS values between baseline and sixth month comparison ( 7.0 at baseline and 6.0 at sixth month, $\mathrm{p}=0.006$ ). Baseline-first month and first-sixth months comparisons were not significant $(\mathrm{p}=0.165$ and $\mathrm{p}=0.106$, respectively). For ODI, no significant change was observed between baseline-first month, first-sixth months, and baseline-sixth month comparisons $(\mathrm{p}=0.109, \mathrm{p}=0.857$, and $\mathrm{p}=0.095$, respectively). For lumbar flexion, all comparisons resulted in significant increase $\left(50.0^{\circ}\right.$ for baseline, $60.0^{\circ}$ at first month, and $70.0^{\circ}$ at sixth month; $\mathrm{p}<0.001$ for baseline-first month comparison, $\mathrm{p}<0.001$ for baseline-sixth month comparison, and $\mathrm{p}<0.001$ for first-sixth months comparison).

Conclusion: Our results show that pain is reduced and mobility is improved with US-guided facet joint injections in patients with FBSS.

Keywords: Facet joint injection, failed back surgery syndrome, low back pain, lumbar disc surgery, postoperative pain, spinal surgery, ultrasound-guided.

Failed back surgery syndrome is a challenging condition for surgeons and pain, physical therapy, and rehabilitation specialists etc. It was estimated 
that $20-40 \%$ of the subjects who underwent surgery developed FBSS. ${ }^{[5]}$ Several treatment modalities exist for pain management after spinal surgery including medical treatment, physical therapy, occupational therapy, epidural injections, alternative treatment modalities (acupuncture etc.), and surgery. ${ }^{[2]}$ Surgical revision is avoided since a revision surgery is not associated with improved pain scores and mobility. Better outcomes may be achieved with comprehensive knowledge on FBSS including etiology, prevention, diagnosis, treatment, and follow-up.

Treatment modalities need to be chosen carefully and correctly. Clear indications for revision surgery were reported in the literature (e.g. significant myelopathy or progressive neurological impairment); however, subjects without clear indications are candidates for minimal invasive interventional procedures. ${ }^{[6]}$ Because of their easier use and high success rates, image-guided injections started to be the preferred modalities for multidisciplinary pain management programs in chronic low back pain (CLBP) ${ }^{[7]}$ Among the image-guided methods, use of ultrasound (US) has been rapidly increasing which allows real-time identification of anatomical structures and improves procedural success.

Although widely evaluated in the literature, the exact mechanism of FBSS is unknown. ${ }^{[8]}$ Facet joints share the load of spine in conjunction with lumbar discs and serve as a potential pain generator after any lumbar spinal surgery. ${ }^{[9]}$ This is why surgery may provoke the degeneration process of facet joints. This report presents a series of patients who underwent US-guided facet injection with lidocaine-betamethasone mixture for FBSS. Thus, in this study, we aimed to evaluate whether US-guided facet joint injection reduces pain and improves mobility in patients with FBSS.

\section{PATIENTS AND METHODS}

This retrospective study was conducted in the Physical Medicine and Rehabilitation and Neurosurgery Clinics of Bakırköy Dr. Sadi Konuk Training and Research Hospital, Istanbul, Turkey. Twenty-seven patients (8 males, 19 females; mean age $43.6 \pm 11.5$ years; range, 31 to 54 years) who underwent an operation for LDH between January 2017 and January 2019 were evaluated. Patients with postoperative FBSS, over the age of 18 years, with a history of chronic function-limiting low back pain with or without lower extremity pain of at least six months (postoperatively), who were capable to understand the study protocol and provide voluntary written informed consent and participate in the outcome measurements, and those with at least six months of follow-up were included. For inclusion, previous surgery should have been performed at least six months ago and no more duration than one year should have passed since surgery. The study protocol was approved by the Bakırköy Dr. Sadi Konuk Training and Research Hospital Ethics Committee (assigned number: 2019/476). A written informed consent was obtained from each patient. The study was conducted in accordance with the principles of the Declaration of Helsinki.

Patients with a positive response to controlled comparative local anesthetic blocks, uncontrollable or unstable opioid use, uncontrolled psychiatric disorders, uncontrolled medical illness, either acute or chronic, any conditions that could interfere with the interpretation of the outcome assessments, pregnant or lactating females, patients with a history or potential for adverse reaction(s) to local anesthetic or steroids, and those with less than six months of follow-up were excluded.

Patients underwent a pre-enrollment evaluation, which included demographic data, medical and surgical history with co-existing disease(s), radiologic investigations, physical examination, work status, opioid intake, pain rating scores using the Numeric Rating Scale (NRS), and functional status assessment using the Oswestry Disability Index (ODI) version 2.0.

A single physician performed the facet joint injection procedures in a sterile ambulatory surgery room. Each facet injection was performed at one level above, same level, and one level below the previous surgical level. The procedures utilized US (Esaote MyLab 60 3-5 MHz convex transducer, Genova, Italy) with participants in the prone position. After sterile preparation, the transducer was placed at axial position on the target area at the lumbar region. Transducer was moved caudally to find the facet joint and the injection was performed with a 22-gauge $60-\mathrm{mm}$ needle $(9 \mathrm{~mL}$ of lidocaine mixed with $6 \mathrm{mg}$ of non-particulate betamethasone [Diprospan flacon, Merc Sharp \& Dohme, New Jersey, USA]) via an in-plane technique. Injection volume was $1 \mathrm{~mL}$. Injections were performed two times once a week. Patients were observed for one hour after the procedure to detect any allergic reactions or weakness in the lower limb.

Multiple outcome measures were utilized which included the NRS (0-10 scale) pain scale, the ODI on a 0-50 scale, and employment status. These measurements were taken at the first and sixth 


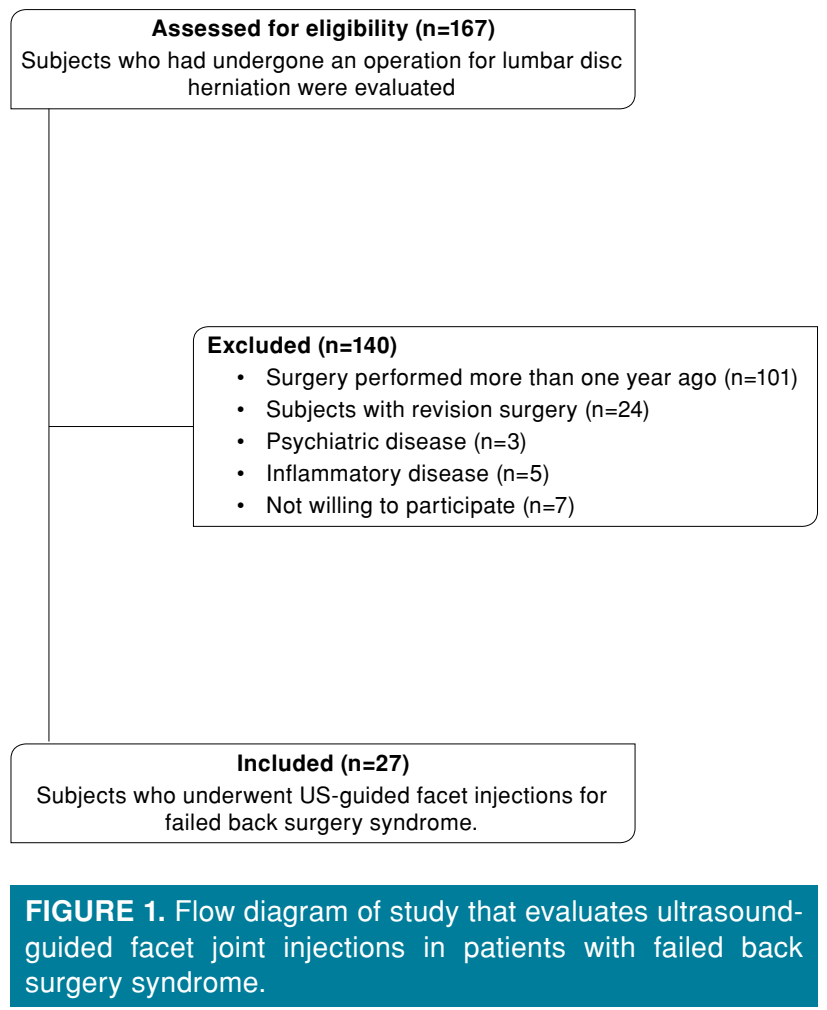

months after the facet injections. The NRS represents no pain with a 0 and the worst pain imaginable with a 10. The ODI was utilized for functional assessment. The value and validity of the NRS and ODI were established. ${ }^{[5]}$ Thresholds for the minimum clinical important difference for the ODI varied from a 4 to 15 point change from of a total score of 50 . However, recent debate on minimally clinical important difference has illustrated that a 50\% reduction in ODI provides a robust measure. ${ }^{[5,8]}$ Thus, significant pain relief and reduced disability status were described as a $50 \%$ or more reduction in scores from baseline.

\section{Statistical analysis}

Data were analyzed using the IBM SPSS Statistics version 22.0 software (IBM Corp., Armonk, NY, USA). Descriptive statistical methods (minimum, maximum, standard deviation, mean, median, frequency, and percentage) were used. The scale scores were not normally distributed according to Shapiro-Wilk test. Therefore, we used the Wilcoxon signed-rank test to assess changes in measurements before and after the intervention. For the analysis, a $p$ value less than 0.05 was considered to be statistically significant.

\begin{tabular}{|c|c|c|c|}
\hline \multicolumn{2}{|c|}{$\begin{array}{l}\text { Demographic data of patients who underwent ultrasound-guided facet } \\
\text { joint injections for failed back surgery syndrome }(n=27)\end{array}$} & & \\
\hline & $\mathrm{n}$ & $\%$ & Mean $\pm S D$ \\
\hline Age (year) & & & $43.6 \pm 11.5$ \\
\hline \multicolumn{4}{|l|}{ Sex } \\
\hline Female & 19 & 70.3 & \\
\hline Male & 8 & 29.7 & \\
\hline Body mass index $\left(\mathrm{kg} / \mathrm{m}^{2}\right)$ & & & $32.5 \pm 4.6$ \\
\hline Duration of pain (month) & & & $6.7 \pm 5.7$ \\
\hline \multicolumn{4}{|l|}{ Low back pain distribution } \\
\hline Bilateral & 18 & 66.6 & \\
\hline Unilateral & 9 & 33.4 & \\
\hline $\begin{array}{l}\text { Mean period between operation } \\
\text { and injection (month) }\end{array}$ & & & $7.8 \pm 1.3$ \\
\hline \multicolumn{4}{|l|}{ Surgery level } \\
\hline One level & 23 & 85.2 & \\
\hline \multirow[t]{2}{*}{ Multilevel } & 4 & 14.8 & \\
\hline & & & Injection level \\
\hline \multicolumn{4}{|l|}{ Surgical level } \\
\hline L3-4 & 3 & 11.1 & (L2-3, L3-4, L4-5) \\
\hline L4-5 & 8 & 29.6 & (L3-4, L4-5, L5-S1) \\
\hline L5-S1 & 12 & 44.4 & (L4-5, L5-S1) \\
\hline Multilevel (L4-5 and L5-S1) & 4 & 14.8 & (L3-4, L4-5, L5-S1) \\
\hline SD: Standard deviation. & & & \\
\hline
\end{tabular}




\begin{tabular}{|c|c|c|c|c|c|c|c|c|c|}
\hline Numeric Rating Scale, & westry D & $\begin{array}{l}\text { ability Index } \\
\text { injections fo }\end{array}$ & $\begin{array}{l}\text { TA } \\
\text { failed bac }\end{array}$ & $\begin{array}{l}\text { LE II } \\
\text { flexion char } \\
\text { surgery syn }\end{array}$ & $\begin{array}{l}\text { es before } \\
\text { rome }(n=2\end{array}$ & and after ul & asound-c & uided fac & t joint \\
\hline & \multicolumn{2}{|c|}{ Baseline } & \multicolumn{2}{|c|}{$1^{\text {st }}$ month } & \multicolumn{2}{|c|}{$6^{\text {th }}$ month } & \multirow[b]{2}{*}{$p^{\mathrm{a}}$} & \multirow[b]{2}{*}{$p^{\mathrm{b}}$} & \multirow[b]{2}{*}{$p^{c}$} \\
\hline & Median & Min-Max & Median & Min-Max & Median & Min-Max & & & \\
\hline Numeric Rating Scale & 7.0 & $5.0-9.0$ & 7.0 & $5.0-9.0$ & 6.0 & $5.0-9.0$ & 0.165 & 0.106 & 0.006 \\
\hline Oswestry Disability Index & 36.0 & $28.0-44.0$ & 34.0 & $28.0-44.0$ & 34.0 & $28.0-42.0$ & 0.109 & 0.857 & 0.095 \\
\hline Lumbar flexion (degree) & 50.0 & $35.0-60.0$ & 60.0 & $45.0-70.0$ & 70.0 & $45.0-85.0$ & $<0.001$ & $<0.001$ & $<0.001$ \\
\hline
\end{tabular}

\section{RESULTS}

Flow diagram of the study was presented in Figure 1. Nearly half of the patients were current workers $(44.3 \%)$. The median duration of time after surgery was $7.8 \pm 1.3$ (range, 6 to 12) months. Previous surgery was performed at one level in $23(85.2 \%)$ and multilevel in four $(14.8 \%)$ patients. Distribution of surgical levels included lumbar L3-4 $(\mathrm{n}=3,11.1 \%)$, lumbar L4-5 ( $\mathrm{n}=8,29.6 \%)$, lumbar L5-S1 $(\mathrm{n}=12$, 44.4\%), and multilevel (lumbar L4-5 and lumbar L5-S1, $\mathrm{n}=4,14.8 \%$ ), respectively. Pain distribution was bilateral in $18(66.6 \%)$ and unilateral in nine (33.4\%) patients. Mean duration of pain was $6.7 \pm 5.7$ months (range, 6 to 12 months) (Table I).

In all patients, successful facet joint injections were achieved without any complications. Lumbar flexion, NRS, and ODI scores were shown in Table II. There was a significant decrease in NRS scores between baseline and sixth month comparison. Numeric Rating Scale score was 7.0 (range, 5.0 to 9.0) at baseline, 7.0 (range, 5.0 to 9.0) at first month, and 6.0 (range, 5.0-9.0) at sixth month ( $p=0.165$ for baseline-first month, $p=0.106$ for firstsixth months, and $\mathrm{p}=0.006$ for baseline-sixth month comparisons, respectively).

The ODI scores were 36.0 (range, 28.0 to 44.0 ) at baseline, 34.0 (range, 28.0 to 44.0 ) at first month, and 34.0 (range, 28.0 to 42.0 ) at sixth month. No significant difference existed for any of the comparisons $(p=0.109$ for baseline-first month, $\mathrm{p}=0.857$ for first-sixth months, and $\mathrm{p}=0.095$ for baseline-sixth month comparisons, respectively).

A significant increase was observed for lumbar flexion. Lumbar flexion degrees were $50.0^{\circ}$ (range, 35.0 to 60 ) at baseline, $60.0^{\circ}$ (range, 45.0 to 70.0 ) at first month, and $70.0^{\circ}$ (range, 45.0 to 85.0 ) at sixth month $(p<0.001$ for baseline-first month, $p<0.001$ for firstsixth months, and $\mathrm{p}<0.001$ for baseline-sixth month comparisons, respectively).

\section{DISCUSSION}

The most important finding of our study is that pain is reduced and mobility is improved with US-guided facet joint injections in patients with FBSS, ${ }^{[10]}$ which is a challenging situation since any part of the back with a nerve supply can be the source of pain including vertebral discs, vertebrae itself, sacroiliac/ facet joints, muscles, and ligaments etc.

It is important to evaluate the patients in a multidisciplinary manner since FBSS has a multifactorial etiology. There are suggestive factors related to the patients such as smoking, obesity, workers' decompensation, and previous psychiatric disorders like depression. ${ }^{[5]}$ There are also factors related with the spinal area such as disc degeneration, foraminal stenosis, epidural fibrosis, adhesive arachnoiditis, and neuropathy. ${ }^{[8]}$ However, the etiology is more likely to be multifactorial and an exact mechanism could not be found. In surgical view, pre-, intra-, and postoperative factors affect the occurrence of FBSS. ${ }^{[6]}$ It is also well known that chronic low back pain may be discogenic, facet joint, sacroiliac joint or other sources related pain. Facet joints are paired joints responsible for stabilization and guide motion of the spine. ${ }^{[8]}$ Among the patients with CLBP, facet joints would be responsible for 15 to $45 \%$ of the pain. ${ }^{[1]}$

Treatment strategy starts with conservative management, physical therapy, and medication. Second line treatments include minimally invasive treatment modalities which are injections, adhesiolysis, radiofrequency ablation, and neuromodulation. Surgery should be avoided and needs to be performed in certain circumstances as profound motor weakness or neurological deficit etc. ${ }^{[5]}$ In a systematic review, Cho et al..$^{[8]}$ reported the adhesiolysis or spinal cord stimulation as the first line treatment of FBSS followed by epidural injections. 
Lumbar facet joints have an intensive nociceptor and nerve supply. ${ }^{[12]}$ They are regarded as one of the potential causes of CLBP. ${ }^{[5,13]}$ Facet joints are responsible for pain and mobility reduction in varying frequency in both CLBP and FBSS. Plain radiography and magnetic resonance imaging (MRI) are the main radiological modalities of which contrast enhanced MRI gives much detailed information for etiological evaluation. ${ }^{[6]}$ However, standard diagnostic tools as medical history, physical examination as well as imaging modalities have low diagnostic accuracy. It was proposed that diagnostic blocks would be much useful for diagnosis of actual pain generator including facet joint originated pain. In the presence of facet joint originated pain, intraarticular injections, nerve blocks or necrolysis of facet joint nerves will be useful. ${ }^{[3]}$

In the present study, a mixture of lidocainebetamethasone was used for facet injections. The volume for facet joint injections changed between 1 and $2 \mathrm{~mL}$. In our study, injection volume was $1 \mathrm{~mL}$. In general, for injection, use of a mixture of a longacting corticosteroid and a local anesthetic is the most common method. ${ }^{[14]}$ Although different injections were used (saline, dextrose, hyaluronic acid, and local anesthetic alone), there is no comprehensive data for their use as corticosteroid/local anesthetic combination. Corticosteroids have anti-inflammatory and anti-edematous action inhibiting neural transmission within the $\mathrm{C}$ fibers. ${ }^{[15]}$ Intraarticular injections have a superiority over intramuscular injections with limited side effects.

Our study used US guidance for facet joint injections. Use of US guidance achieved $100 \%$ success rate without any complications. ${ }^{[10]}$ Initial injections were performed with landmark technique; however, without using an additional imaging modality (fluoroscopy, US guidance etc.), landmark technique may not provide adequate anesthesia while it increases the complications such as hemorrhage, dural perforation, and incorrect adjacent tissue injections. ${ }^{[7,16]}$ Incorrect injections may be high as $14-56 \%$ without an additional imaging modality. ${ }^{[16]}$

Fluoroscopy, US guidance, computed tomography (CT), and MRI are the options for injection guidance. ${ }^{[15]}$ Use of any guidance affects the technical success, increases clinical efficacy, and decreases complication rates. ${ }^{[15,16]}$ Fluoroscopy guidance is one of the most commonly used methods for guidance; however, fluoroscopy needed an additional team, increased the costs, and patients received a degree of radiation. With the use of fluoroscopy, the average direct exposure dose will be between $0.4-4.0 \mathrm{rad} / \mathrm{min}^{.16]}$

Recently, US guidance has been proven to have efficacy in lumbar paravertebral region. Instead of linear probes, low frequency probes need to be selected since they have much lower resolution. They allow a direct, reliable, safe, and accurate technique as well as visualization of the target tissue while enabling real-time needle guidance. Spread of local anesthetics will be visible while procedure time decreases. Unlike fluoroscopy and $\mathrm{CT}$, radiation exposure should be avoided and the operation time decreased. ${ }^{[7]}$ Patients with obesity may negatively affect the results of US guidance.

Akkaya et al. ${ }^{[16]}$ compared US- and fluoroscopyguided caudal epidural steroid injections in postlaminectomy patients. In 30 patients, results indicated faster block time with US-guided injection with similar improvement in low back pain and functions. Obernauer et al. ${ }^{[13]}$ compared US-guided facet joint injections with CT-guided facet joint injections. In 40 patients, US-guided facet joint injections achieved $100 \%$ success rate with significantly faster needle placement $(04: 46 \mathrm{~min}$ for US guidance versus 11:12 min for CT guidance for one-level and 05:49 min for US guidance versus 14:32 min for CT guidance for two-level injections). Both groups demonstrated similar pain relief and US guidance resulted with less radiation dose exposure than CT group $\left(27.6 \mathrm{mGy}^{*} \mathrm{~cm}\right.$ for US guidance versus $88.2 \mathrm{mGy}^{*} \mathrm{~cm}$ for CT guidance for one-level and $32.5 \mathrm{mGy}^{*} \mathrm{~cm}$ for US guidance versus $205.0 \mathrm{mGy}^{*} \mathrm{~cm}$ for CT guidance for two-level injections).

Despite correct facet injections, pain relief could not be observed. ${ }^{[3]}$ In our study, injections to facet joins significantly decreased pain and improved mobility without complications. When evaluating results of an intervention, the decision should also be focused on the range of motion as well as pain intensity. A recent meta-analysis also noted that the intraarticular facet joint injections have no complications except a little discomfort. However, this meta-analysis noted that studies reporting positive outcomes are limited and the effectiveness of facet joint injections needs to be further studied.$^{[12]}$ In a prospective trial including 18 patients, Kawu et al. ${ }^{[11]}$ reported better outcomes with the use of intraarticular facet joint injections (bupivacaine-methylprednisolone combination) than physiotherapy alone. Oswestry Disability Index, visual analog scale (VAS), and patient satisfaction scores were significantly better in facet 
injection group. Çelik et al. ${ }^{[17]}$ reported a comparative study on 80 patients. Zygapophysial joints blockage (prilocaine, bupivacaine, and methylprednisolone acetate) was compared with conservative management (bed rest, diclofenac sodium, and thiocolchicoside). Patients were evaluated with ODI and VAS. Results indicated that facet joint injections demonstrated faster and greater decrease in pain levels compared with conservative management.

In contrast, Carette et al. ${ }^{[18]}$ compared steroid injection (methylprednisolone acetate, $n=49$ ) with isotonic injection (isotonic saline, $\mathrm{n}=48$ ). All patients reported relief of pain after injections of local anesthetic into the facet joints. In terms of pain severity, back mobility, and functional impairment, groups did not differ at first and third months. At sixth month control, methylprednisolone group demonstrated better pain scores and less disability indicating a little benefit from steroid injections for facet joint injections. In an updated Cochrane review, Staal et al. ${ }^{[19]}$ reviewed studies that reported the results of injections for subacute or chronic low back pain. Results of 18 trials (1,179 patients) that used injections for epidural sites and facet joints or local sites indicated that evidence is not sufficient to support the use of injection therapy in subacute and CLBP. However, authors also noted that every subgroup of patients needs to be evaluated separately since specific groups may respond to specific types of injections.

The main limitations of our study are the retrospective design and the lack of a control group. Moreover, due to our study's relatively short followup duration, we were not able to assess the long-term effects of injections.

In conclusion, treatment of patients with FBSS is challenging. Among the interventional pain management procedures, we believe that facet joint injection serves as an effective treatment option particularly in selected cases. Our results showed reduced pain and improved mobility in patients with FBSS in short term. Combination for steroid-local anesthetic under US guidance resulted in good success with no observed complications. Comparative studies with different treatment modalities may provide the optimal treatment modality in FBSS.

\section{Declaration of conflicting interests}

The authors declared no conflicts of interest with respect to the authorship and/or publication of this article.

\section{Funding}

The authors received no financial support for the research and/or authorship of this article.

\section{REFERENCES}

1. Gu J, Guan F, Zhu L, Guan G, Chi Z, Wang H, et al. Risk Factors of Postoperative Low Back Pain for Lumbar Spine Disease. World Neurosurg 2016;94:248-54.

2. von der Hoeh NH, Voelker A, Gulow J, Uhle U, Przkora $\mathrm{R}$, Heyde CE. Impact of a multidisciplinary pain program for the management of chronic low back pain in patients undergoing spine surgery and primary total hip replacement: a retrospective cohort study. Patient Saf Surg 2014;8:34.

3. Civelek E, Cansever T, Kabatas S, Kircelli A, Yilmaz C, Musluman M, et al. Comparison of effectiveness of facet joint injection and radiofrequency denervation in chronic low back pain. Turk Neurosurg 2012;22:200-6.

4. Choy DS. Percutaneous laser disc decompression (PLDD): 352 cases with an 8 1/2-year follow-up. J Clin Laser Med Surg 1995;13:17-21.

5. Baber Z, Erdek MA. Failed back surgery syndrome: current perspectives. J Pain Res 2016;9:979-87.

6. Hussain A, Erdek M. Interventional pain management for failed back surgery syndrome. Pain Pract 2014;14:6478.

7. Loizides A, Peer S, Plaikner M, Spiss V, Galiano K, Obernauer $\mathrm{J}$, et al. Ultrasound-guided injections in the lumbar spine. Med Ultrason 2011;13:54-8.

8. Cho JH, Lee JH, Song KS, Hong JY, Joo YS, Lee DH, et al. Treatment Outcomes for Patients with Failed Back Surgery. Pain Physician 2017;20:E29-E43.

9. Lim ST, Kim WJ, Lee SH, Min SH, Jung B. Changes in facets and adjacent discs following lumbar artificial disc replacement arthroplasty. Joint Dis Rel Surg 2005;16:118-24.

10. Atik OŞ. Which articles do we prefer to publish? Eklem Hastalik Cerrahisi 2018;29:1.

11. Kawu AA, Olawepo A, Salami AO. Facet joints infiltration: a viable alternative treatment to physiotherapy in patients with low back pain due to facet joint arthropathy. Niger J Clin Pract 2011;14:219-22.

12. Vekaria R, Bhatt R, Ellard DR, Henschke N, Underwood M, Sandhu H. Intra-articular facet joint injections for low back pain: a systematic review. Eur Spine J 2016;25:1266-81.

13. Obernauer J, Galiano K, Gruber H, Bale R, Obwegeser AA, Schatzer R, et al. Ultrasound-guided versus Computed Tomography-controlled facet joint injections in the middle and lower cervical spine: a prospective randomized clinical trial. Med Ultrason 2013;15:10-5.

14. Pirbudak L, Karakurum G, Şatana T, Karadaşli H, Topalhan M, Öner Ü, et al. Epidural steroid injection and amitriptyline in the management of acute low back pain originating from lumbar disc herniation. Jt Dis Relat Surg 2003;14:89-93.

15. Filippiadis DK, Kelekis A. A review of percutaneous techniques for low back pain and neuralgia: current trends in epidural infiltrations, intervertebral disk and facet joint therapies. Br J Radiol 2016;89:20150357.

16. Akkaya T, Ozkan D, Kertmen H, Sekerci Z. Caudal epidural steroid injections in postlaminectomy patients: comparison of ultrasonography and fluoroscopy. Turk Neurosurg 2017;27:420-5.

17. Celik B, Er U, Simsek S, Altug T, Bavbek M. Effectiveness of lumbar zygapophysial joint blockage for low back pain. Turk Neurosurg 2011;21:467-70. 
18. Carette S, Marcoux S, Truchon R, Grondin C, Gagnon J, Allard $\mathrm{Y}$, et al. A controlled trial of corticosteroid injections into facet joints for chronic low back pain. N Engl J Med 1991;325:1002-7.
19. Staal JB, de Bie RA, de Vet HC, Hildebrandt J, Nelemans P. Injection therapy for subacute and chronic low back pain: an updated Cochrane review. Spine (Phila Pa 1976) 2009;34:49-59. 